\title{
Transatlantica
}

Revue d'études américaines. American Studies Journal

$1 \mid 2002$

Jeune République

\section{Biographie et histoire dans la jeune République.}

Réflexions méthodologiques

\section{Naomi Wulf}

\section{CpenEdition}

Journals

Édition électronique

URL : http://journals.openedition.org/transatlantica/392

DOI : $10.4000 /$ transatlantica.392

ISSN : $1765-2766$

Éditeur

AFEA

Référence électronique

Naomi Wulf, «Biographie et histoire dans la jeune République. », Transatlantica [En ligne], 1 | 2002, mis en ligne le 30 juin 2006, consulté le 29 avril 2021. URL : http://journals.openedition.org/transatlantica/ 392 ; DOI : https://doi.org/10.4000/transatlantica.392

Ce document a été généré automatiquement le 29 avril 2021

\section{(c) (†) $\odot$}

Transatlantica - Revue d'études américaines est mis à disposition selon les termes de la licence Creative Commons Attribution - Pas d'Utilisation Commerciale - Pas de Modification 4.0 International. 


\title{
Biographie et histoire dans la jeune République.
}

\author{
Réflexions méthodologiques
}

Naomi Wulf

Biographie : sorte d'histoire qui a pour objet la vie d'une seule personne Littré, cité par Ferro (85)

1 Depuis 1980, des historiens issus de l'École des Annales cherchent à redonner une place à la biographie dans l'écriture historique. Cette réhabilitation de la biographie historique n'est pas un simple retour aux canons de la biographie telle qu'elle se pratiquait jusqu'alors (héroïque et linéaire). Elle intègre les acquis de l'histoire sociale et culturelle et donne ainsi une importance différente aux acteurs historiques : il n'est plus question de faire simplement l'histoire des grands hommes, mais de regarder le (ou les) acteur(s), célèbres ou non, comme des témoins, des reflets, des révélateurs de leur époque. La biographie n'est plus celle du seul individu; c'est l'histoire d'une époque vue au travers de cet individu (ou groupe d'individus), qui n'est plus présenté comme un héros, mais comme une sorte de réceptacle, au carrefour d'événements, de courants ou de mouvements que son récit de vie rend parfois plus tangibles.

2 Je me propose ici de retracer ce mouvement de retour vers la biographie et de l'évaluer à la lumière des travaux récents qui invitent à la pratique d'une « micro-histoire », loin de la longue durée (Ginzburg), et notamment d'ouvrages récents sur la jeune république américaine. En effet - et c'est que je voudrais discuter ici - cette approche biographique, individuelle ou collective, présente un intérêt tout particulier dans le cadre de l'étude de la jeune république, période qui voit justement naître, avec l'émergence d'une véritable culture de masse démocratique, un sentiment, voire une idéologie, individualiste simultanément théorisée par certains témoins (Cooper, Brownson, Emerson, Tocqueville).

3 Les historiens et les sociologues ont, depuis plus de vingt ans, opéré un retour vers la biographie, mais conçue d'une manière nouvelle. Les années 1950 et 1960 avaient été marquées par un rejet de l'histoire biographique, conséquence de l'émergence de la 
nouvelle histoire sociale dominée par les sciences humaines (Ferro 86). Cette remise en question du mode biographique trouve son aboutissement en sociologie au mileu des années 1980 avec «l'Illusion biographique » qui, selon Pierre Bourdieu, reconstruirait une vie selon une logique artificielle, voire « absurde » (Bourdieu 69, Peneff 26).

4 La critique de la biographie par Bourdieu, plutôt que de les en éloigner, a invité les historiens à la regarder sous un angle neuf. Car on assiste à un retour à la biographie, mais qui n'est en rien un retour à l'histoire des "grands hommes ", à la biographie «traditionnelle, superficielle, anecdotique, platement chronologique, sacrifiant à une psychologie désuète, incapable de montrer la signification historique générale d'une vie individuelle» (Le Goff 1989, 50). L'introduction du genre biographique dans l'historiographie actuelle est au contraire un outil supplémentaire apporté à la Nouvelle Histoire, c'est « une façon de continuer à faire de l'histoire par d'autres moyens ", comme l'a expliqué à maintes reprises Le Goff lors de ses recherches sur Saint Louis (1992).

5 Ainsi dans la tradition de l'esprit des Annales, la biographie démarre sur une "question" et se formule comme un cas d'« histoire-problème" (Le Goff 50-1; Braudel). Comme tout récit de vie, elle demande néanmoins de se soumettre à une chronologie d'événements, mais contrairement à la vie-destin, c'est « une construction faite de hasards, d'hésitations, de choix", qui permet d'échapper à l'illusion biographique (Le Goff 1996, 18).

6 Alors que Fernand Braudel a préconisé la «longue durée» dans toute analyse historique, Le Goff insiste sur le temps d'une vie individuelle comme «durée significative pour l'histoire » (Le Goff 1989, 51) et invite plus généralement à chercher des "durées pertinentes en histoire ", "qui permettent non seulement d'observer un phénomène », mais aussi de voir "une grande partie de l'évolution historique » (1992, 40). Selon lui, la vie humaine est un très bon exemple en histoire et la vie de Saint Louis, en particulier, "mesure" toute une série de phénomènes (40-41). En outre, cette approche présente l'intérêt de surmonter la fausse opposition entre histoire narrative et histoire structuraliste $(1996,18)$ : Saint Louis nous renseigne sur les mécanismes fondamentaux de son époque, c'est-à-dire qu'en étudiant Saint Louis, on retrouve les structures par un autre biais, "presqu'en action et en incarnation", loin des froides structures (1992, 41-2, 43).

7 La biographie libère aussi de la fausse opposition entre individu et société : l'individu n'existe pas seul, il n'« existe que dans un réseau de relations sociales diversifiées » (1996). Car dans la vie d'un individu, convergent événements et forces sociales (Lepore 131), comme convergent l'individu, ses idées, et le contexte social auquel il appartient. Dans son étude récente sur les Marannes, Nathan Wachtel démontre justement, dans ce qu'il qualifie d'" enquête micro-historique ", en quoi chaque individu étudié est situé dans une trajectoire donnée ainsi que dans « son rapport avec les autres au sein de la société globale [...] ». Les individus « représentent bien plus qu'eux-mêmes et chacun à sa manière exprime quelque chose du collectif dont ils ne peuvent s'abstraire » (30-33).

8 La biographie permettrait ainsi l'approche de ce que Emmanuel Le Roy Ladurie, après Braudel, appelle «l'histoire». Ceci est paradoxalement rendu possible par le biais de l'étude d'un individu qui n'est pas nécessairement illustre, et peut-être justement parce qu'il ne l'est pas : «Car les destins individuels sont situés dans les divers réseaux où ils s'entrecroisent : la domus, l'espace régional, l'univers spirituel ; le milieu et l' 'outillage 
mental' de l'époque. [...] Le singulier ouvre sur le général dans la mesure où il est restitué dans son inépuisable richesse » (Wachtel et Valensi 8).

Biographie et histoire intellectuelle, culturelle et sociale. Convergences

transatlantiques

9 La biographie résoudrait alors le difficile rapport entre histoire intellectuelle et histoire sociale. C'est notamment sur ce point qu'on trouve une convergence entre l'approche historiographique française et américaine. En effet, les théories relativement récentes sur la biographie qui émanent essentiellement de chercheurs français issus des Annales rencontrent des questions posées par des historiens américains, et notamment par les historiens de la jeune république. Ce sont ces mêmes questions qui se sont imposées à moi depuis que j'ai entrepris des recherches sur la démocratie jacksonienne au détour du parcours et des écrits de Orestes Brownson, 1803-1876 (Wulf).

Les théories françaises récentes rejoignent en effet certaines préoccupations historiographiques américaines, et ceci selon deux axes principaux: celui des mutations à l'intérieur même de l'histoire intellectuelle et celui de l'émergence de l'histoire culturelle. Or il semble bien qu'une nouvelle conception de l'histoire biographique puisse être en mesure d'offrir une réponse à certains questionnements qui ont animé la communauté historique américaine depuis les années 1960.

11 D'une part, l'approche biographique permet de résoudre la contradiction qui a été longtemps soulignée entre histoire des idées et histoire sociale, et qui a notamment nourri d'importants débats au sein de l'historiographie de la Révolution américaine (voir les débats qui ont opposé historiens sociaux et historiens républicanistes sur le rôle des idées comme causes ou motivations dans les actions révolutionnaires; voir les synthèses de Shalhope ou Gordon). Dans un ouvrage de 1979 devenu un classique, John Higham et Paul Conkin incitent les historiens à faire une " histoire sociale des idées " (xii), où les idées de l'individu étudié ne le seraient pas en tant que "motif » ou "cause » des événements mais en tant que "mode de représentation d'un moment historique ». Il s'agirait donc de trouver un équilibre entre l'individu, son libre-arbitre, ses intentions personnelles et son interaction avec l'échelle plus grande des conventions culturelles et des mentalités collectives (Wood 1979, 38).

D'autre part, parce que, par définition, elle est centrée sur un individu, la biographie pose le problème de la "représentativité » de cet individu (Vesey 20). Or avec le développement de l'histoire culturelle, les approches se sont concentrées en particulier sur les liens entre individu et culture. Dans un article fréquemment cité, David Brion Davis se demande justement comment trouver «les points d'intersection» entre un individu et le développement du cadre social, culturel et politique auquel il participe. Selon lui (et en cela il rejoint l'opinion de Le Goff) la biographie est intéressante parce qu'elle offre un regard concret et un sens du développement de l'histoire qui manquent souvent en histoire culturelle (Davis 705). La biographie collective offre une solution méthodologique possible à ces questions, en ce qu'elle permet l'étude d'un groupe d'individus qui représentent une classe sociale, une profession, un intérêt, pourvu que l'on définisse au préalable avec précision la place qu'occupent dans la structure sociale ces hommes et ces femmes représentatifs (704). On peut également examiner de près la manière dont les crises personnelles d'un individu complexe reflètent les tensions dans la culture environnante et comment les résolutions personnelles du conflit par l'individu font écho aux transformations de cette même culture (704). 

me propose d'examiner dans le cadre de la période qui nous intéresse ici, à travers quelques exemples qui nous permettront de retrouver les termes du débat théorique. Quelques historiens récents de la période jacksonienne, en particulier, ont justement insisté sur la nécessité dans l'étude de la " démocratie jacksonienne » de s'arrêter sur des parcours individuels, à une époque où les acceptions même d'individu, d'individualisme et d'individualité subissent des bouleversements (Watts xxi, Howe 4, Feller 135-162). Si on en croit le thème choisi pour le dernier Congrès de la Society for the History of the Early American Republic : «Lived Lives in the Early Republic » (SHEAR, juillet 2001), les historiens de la jeune république en général se posent manifestement ces questions.

Biographies collectives dans la jeune république

Telle est l'approche préconisée par Steven Watts et Daniel Walker Howe, deux historiens qui analysent la transformation de la culture politique, d'une part entre 1790 et 1820 lors de l'émergence d'une « culture capitaliste » (Watts), et d'autre part, dans la période qui suit 1834, avec la fondation du parti whig (Howe). Dans ce qu'ils appellent chacun une biographie collective de deux groupes idéologiques centraux à la jeune république, Watts et Howe se réclament de la même méthodologie biographique qu'ils citent tous deux en introduction :

By showing how cultural tensions and contradictions may be internalized, struggled with, and resolved within actual individuals, biography offers the most promising key to the synthesis of culture and history (Davis 705).

Ils aboutissent en effet, à travers ces deux collections de portraits organisées de manière thématique à appréhender des aspects centraux du libéralisme politique de la jeune nation, grâce à l'étude du rôle joué par des personnalités politiques importantes (Calhoun ou Webster, pour n'en citer que deux, ainsi que des théoriciens de l'économie politique comme Daniel Raymond), de leurs idées et de leur pratique politique. Watts et Howe s'intéressent à des personnages clés qui leur permettent d'éclairer deux moments de transition fondamentaux pour la compréhension de l'histoire de la jeune république. Dans le premier cas, Watts analyse l'émergence du libéralisme à l'occasion de la guerre de 1812 et établit une convergence entre la guerre et l'élaboration de cette nouvelle idéologie libérale qui vient prendre la place d'un républicanisme jusque là hégémonique. Howe éclaire, quant à lui, au travers de cette galerie de portraits d'hommes politiques whigs (tels Henry Clay ou Horace Mann), la création d'un second parti face au jacksonisme dominant et plus fréquemment étudié. Dans les deux cas, les grandes figures de ces mouvements sont placées dans un cadre qui intègre la dimension culturelle de l'époque, y compris la dimension religieuse (Howe ; voir Bergamasco dans ce même numéro).

La mise en évidence d'une époque charnière est particulièrement explicite dans deux autres études faites par les historiennes Jean Baker et Joyce Appelby, qui proposent également des biographies collectives, mais dont l'unité est clairement la génération et non plus le groupement politique : il s'agit, dans les deux cas, de la présentation de la culture politique et sociale propre à la génération née juste après la Révolution ou à celle qui était trop jeune pour y avoir participé directement. Chez Baker, ce sont les jacksoniens du Nord, qui sans avoir pris part à la Révolution en personne, ont néanmoins baigné dans une culture post-révolutionnaire. Cette culture imprégna toute une génération d'hommes politiques, qui sont à l'origine de la formation du parti 
démocrate autour du Président Jackson - depuis George Bancroft jusqu'à Martin Van Buren. Du fait de leur lien générationnel, ces nouveaux hommes politiques entretiennent un rapport spécifique au passé révolutionnaire et partagent des valeurs communes qui deviennent centrales à l'idéologie du parti auquel ils appartiennent, comme le refus de la politique partisane, la tradition républicaine de méfiance à l'égard du pouvoir, ou la peur républicaine du complot. Il en ressort un portrait de groupe qui véhicule une culture politique commune, mais dont l'approche pose néanmoins la question de la différence partisane avec les whigs qui pourtant appartiennent à la même génération.

17 L'approche de Appleby est moins strictement politique. Encore plus que Baker, elle met en scène ce qu'elle appelle la première génération d'Américains, à partir de 200 autobiographies (certaines sont publiées dans un recueil séparé ; Appleby 1997). Il en ressort la biographie collective d'une génération prise entre la Révolution et la période jacksonienne, laquelle est justement rarement présentée par elle-même alors même qu'elle a le sentiment d'être la "première » (25). Cette première génération d'Américains apparaît à travers les témoignages de personnes célèbres et de personnages moins illustres, dont les vies dans la jeune république sont retracées selon des rubriques diverses qui constituent les différents chapitres de l'ouvrage ("enterprise»-typique d'une période justement dominée par l'entreprise individuelle avec l'émergence d'une culture capitaliste - «careers ", " distinctions ", " intimate relations ", " reform », " a new national identity »). Appleby donne ainsi de l'importance dans toute leur diversité à des individus qui participent par leurs trajectoires uniques au processus qui transforme des sociétés coloniales encore distinctes et diverses en une seule nation américaine-chapitre qu'elle garde volontairement pour la fin de l'ouvrage. En effet, à travers ses témoignages individuels, l'auteur ne voit pas transparaître de sentiment national. Elle choisit plutôt de décrire une nation en construction à partir de l'ensemble de ces expériences uniques.

Au-delà de certaines critiques qu'on pourrait faire - car l'Amérique est décrite selon le cliché libéral d'un peuple d'individus entreprenants, toujours en mouvement, à la Tocqueville ou à la Wiebe, avec le sentiment que cette expérience est particulière à l'Amérique (Appleby 25, Waldstreicher, Johnson) -, ce qui est frappant c'est le changement d'optique chez cette historienne du libéralisme jeffersonien et de l'émergence du capitalisme : elle est passée de l'analyse strictement idéologique de ses ouvrages précédents (Appleby 1984, 1992), à l'incarnation de cette idéologie à travers les 200 parcours individuels qu'elle a suivis ${ }^{1}$.

Toutefois, malgré l'appellation de biographie collective, il ne ressort pas de ces quatre exemples une approche véritablement biographique, au sens du récit de vie. Il s'agirait plutôt de galeries de portraits organisées selon des principes différents, mais qui présenteraient néanmoins la spécificité de vouloir photographier une époque charnière (que ce soit la période jeffersonienne ou jacksonienne); l'expérience individuelle y remplace l'étude plus classique des comportements collectifs (nationaux ou partisans) qui ont longtemps occupé la première place dans les débats historiographiques, ce qui est tout particulièrement vrai de la période jacksonienne (voir synthèse de Feller).

L'individu dans la jeune république Qu'en est-il alors de la place de l'individu seul, dans l'histoire et l'historiographie de la jeune République? Je souhaiterais me concentrer sur deux approches qui me paraissent rejoindre au mieux les préoccupations méthodologiques exprimées plus haut. 
L'ouvrage de Mary Cayton sur Emerson, découvert en cours de thèse, m'avait permis de confirmer un certain nombre d'hypothèses de travail ; et l'ouvrage de Alan Taylor sur le père de James Fenimore Cooper me paraît une synthèse extrêmement originale de pans fondamentaux de l'historiographie de la jeune République à travers l'étude d'un individu.

21 L'approche de Mary Cayton dans son étude de Ralph Waldo Emerson est justement celle préconisée par Davis et reprise par Watts et Howe, mais appliquée de manière plus convaincante: comment un phénomène culturel, idéologico-culturel, voire philosophique, celui de la montée de l'individualisme dans une démocratie (tant abordé d'un point de vue théorique depuis Tocqueville jusqu'à en devenir un cliché sur la jeune République), peut-il être analysé à travers la vie d'un célèbre essayiste de la Renaissance littéraire américaine ? Il ne s'agit en aucun cas d'une biographie littéraire (Viala), mais bien de la manière dont le parcours d'Emerson, à la fois acteur de son époque, mais également produit de celle-ci et auteur de surcroît, portant un regard d'intellectuel et de philosophe sur ses contemporains et sur les hommes en général, concentrerait simultanément une réaction à la culture de l'individualisme en plein essor et la transmission de cette culture. Cayton propose ainsi une synthèse entre histoire littéraire et histoire sociale tout en se défendant explicitement de faire la biographie d'un individu isolé sur lequel elle aurait superposé le contexte social et historique (ix). Ce qui l'intéresse plutôt, et en cela son livre rejoint le Saint Louis de Le Goff, c'est de voir en quoi Emerson pense et reflète les dilemmes émotionnels provoqués par le nouvel ordre économique et social qu'est le capitalisme émergent de Nouvelle-Angleterre (xi). Emerson se présente dans sa vie et dans son œuvre au confluent de l'organicité de la culture fédéraliste de son Boston natal, centrée sur la déférence et la cohésion sociale, et de la croissance du marché et de ses égoïsmes (7-9). A la charnière entre le déclin de la tradition fédéraliste et l'émergence du libéralisme jeffersonien (compris ici comme l'accès pour tout un chacun au bien public), l'œuvre d'Emerson va tendre vers la recherche de la vertu individuelle, qui n'est pas à confondre avec la poursuite de l'intérêt privé et économique (52). Le paradoxe qui se dessine alors à travers la vie et les écrits d'un auteur qui se veut justement public, en tant qu'orateur et homme de lettres, est qu'il s'emploie à prêcher un ordre moral qui va justement nourrir la philosophie de l'individualisme qu'il rejette dans les mutations sociales bostoniennes. Car son public ne perçoit pas la distinction subtile qu'il développe entre la recherche d'une vertu individuelle et le rejet de l'égoïsme marchand. Emerson aurait, contre son gré, participé à l'invention de la conception moderne de l'individu, fondement du nouvel ordre social dont il appelait justement à se méfier.

Ainsi, l'individu est ici à la fois acteur, plus ou moins critique, et produit de sa période ; et son parcours éclaire la période de deux manières. L'une est explicite, par la démarche volontaire de l'observateur qui propose une analyse critique de la société contemporaine. L'autre se devine dans l'implicite de son parcours qui illustre à son insu les tensions, conflits, et contradictions de son temps, tous essentiels pour la comprehension de la période ; alors, l'individu incarne en quelque sorte ces tensions (240).

23 C'est dans cette dimension de l'implicite que ces récits de vie semblent rejoindre la micro-histoire (Ginzburg) et donner une place au symbolique, voire à la fiction dans le processus d'accès à la connaissance historique. Cette synthèse est réalisée avec brio par 
Alan Taylor dans son ouvrage primé, qui relate l'histoire de la construction de la petite ville de Cooper's Town dans le Nord de l'État de New York, à la fin du XVIII ${ }^{\mathrm{e}}$ siècle, par le père du célèbre romancier américain James Fenimore Cooper. L'histoire de l'ascension de cet homme ambitieux de l'Amérique post-révolutionnaire unit, tout au long de l'ouvrage, trois niveaux d'analyse: la base de départ est le récit biographique qui retrace la vie de William Cooper et de sa famille. Ce récit s'inscrit dans l'histoire sociale et politique de la ville de Cooperstown, dans celle du Comté d'Otsego, de l'État de New York, voire des États-Unis mêmes entre 1786 et 1820 . Ce faisant, Taylor s'inspire de la réinvention littéraire de la construction de la ville par le fils James Fenimore Cooper dans son roman The Pioneers (1823).

Alan Taylor nous offre une très belle leçon d'interdisciplinarité: il nous montre comment une histoire individuelle peut accéder à une échelle plus large en ce qu'elle permet de tirer des conclusions qui intéressent l'histoire de la jeune République dans son ensemble. La démarche est chronologique et dans chaque partie se superposent les trois niveaux interprétatifs sus-mentionnés qui mettent en parallèle trois points de vue qui se complètent : celui de William Cooper lui-même, dans son Guide in the Wilderness qu'il écrit a posteriori sur son expérience (1807); celui des sources plus objectives de l'histoire classique (cartes, cadastres, correspondances, registres divers, ouvrages secondaires) ; et celui de la re-création littéraire par son fils romancier.

On voit notamment en quoi William Cooper symbolise l'histoire de la génération révolutionnaire qui obtient un accès aux terres convoitées, grâce à la défaite d'une famille aristocratique. Cet accès à une terre qu'il croit vierge (Cooper dans son roman introduira des liens sociaux préexistants) fournit l'occasion de faire le récit de l'occupation de la région par des strates successives d'habitants. Puis William Cooper, en architecte du passage d'une propriété privée à un village commercial, illustre la création d'une ville-marché, véritable plaque tournante dans le réseau de production et de vente qui s'amorce alors et présente le passage symbolique, dans la période post-révolutionnaire, d'une société agraire à une société de marché (avec la transformation de la nature, le développement des transports, ou celui de l'élevage faute de ressources minières).

Cette construction s'accompagne d'une ambition politique où les conflits personnels de cette figure volontaire entrent alors en résonnance avec les conflits partisans qui opposent les Fédéralistes aux Républicains dans les années 1790 : dans la tentative de Cooper de faire de la région d'Otsego un comté, Taylor étudie les points d'intersection entre cette ambition toute personnelle et la vie politique plus large (alliances et oppositions avec des personnalités de l'État de New York), mettant ainsi en évidence le rayonnement de ces conflits du local au régional et jusqu'au national. Héros d'une ascension réussie grâce à des entreprises commerciales qui ne masqueront jamais des origines modestes, William Cooper cristallise les hésitations contemporaines entre ambitions artistocratiques et méthodes populaires. Père ou ami du peuple ? William Cooper résume dans ses contradictions propres l'essence de la politique de la jeune République, avec d'une part les Fédéralistes tenants des traditions, de la respectabilité, de la recherche de l'ordre; et d'autre part, son adversaire politique qui représente la tendance plus égalitaire et populiste, voire démagogue, des Républicains qui encouragent l'entreprise individuelle et compétitive en vue d'une réussite personnelle. Toutes ces péripéties politiques et personnelles mènent finalement au déclin de la ville qui revient finalement aux héritiers des propriétaires d'origine : une élite succède ainsi 
à une autre, contrairement au mythe de la république jeffersonienne qui préconisait une redistribution relativement égalitaire de la propriété. Dans la re-création fictionnelle, le fils Cooper dessine un continuum qui va du passé colonial à la jeune République, oblitérant et la rupture révolutionnaire et les conflits politiques qu'il perçoit comme nuisibles à la cohésion communautaire. Taylor, lui, conclut de façon plus pessimiste par l'échec de la jeune République à maintenir la communauté organique idéale.

\section{BIBLIOGRAPHIE}

Appleby, Joyce. Capitalism and a New Social Order. New York : New York UP, 1984.

Appleby, Joyce. Liberalism and Republicanism in the Historical Imagination. Cambridge : Harvard UP, 1992.

Appleby, Joyce. Recollections of the Early Republic Selected Autobiographies. Boston : Northeastern UP, 1997.

Appleby, Joyce. Inheriting the Revolution: The First Generation of Americans. Cambridge : The Belknap Press, Harvard UP, 2000.

Arnaud, Claude. «Le retour de la biographie : d'un tabou à l'autre ». Le Débat 54 (1989) : 40-47.

Braudel, Fernand. « Histoire et sciences sociales. La longue durée », Annales ESC, XIII, 4, 1958 :

725-753. Repris dans Ecrits sur l'histoire. Paris : Flammarion, 1969. 41-83.

Bourdieu, Pierre. «L'Illusion biographique ». Actes de la recherche en sciences sociales 62-63 (1986): 69-72.

Brownson, Orestes. The Works of Orestes Brownson. Ed. Henry F. Brownson. 20 vols. Detroit : Thorndike Nourse, 1882-1887.

Cayton, Mary Cupiec. Emerson's Emergence : Self and Society in the Transformation of New England, 1800-1845. Chapel Hill : University of North Carolina Press, 1989.

Cooper, James Fenimore. The American Democrat. 1838. New York : Penguin, 1989.

Higham, John et Paul Conkin eds. New Directions in American Intellectual History. Baltimore : The Johns Hopkins UP, 1979.

Davis, David Brion. « Some Recent Directions in American Cultural History. » AHR 73 (February 1968) : 696-707.

Diggins, John Patrick. On Hallowed Ground : Lincoln and the Foundations of American History. New Haven and London : Yale UP, 2000.

Emerson, Ralph Waldo. Essays and Lectures. Ed. Joel Porte. New York : The Library of America, 1963.

Esprit 184 (août-sept 1992). Dossier « Quand l'historien se fait biographe » : 29-59. 
Feller, Daniel. « Politics and Society : Toward a Jacksonian Synthesis. » Journal of the Early American Republic Summer 1990 (10) : 135-162.

Ferro, Marc. « La biographie, cette handicapée de l'histoire ». Le Magazine littéraire (avril 1989) : 85-86.

Ginzburg, Carlo. « L'histoire est une fiction qui peut être prouvée ». Le Monde des débats 25 (mai 2001) : 42-45.

Gordon, Colin. «Crafting a Usable Past : Consensus, Ideology and Historians of the American Revolution. » William and Mary Quarterly 46 (October 1989) : 671-695.

Howe, Daniel Walker. The Political Culture of the American Whigs. Chicago : Chicago UP, 1979.

Le Goff, Jacques. « Comment écrire une biographie historique aujourd'hui ». Le Débat 54 (1989) : 48-53.

Le Goff, Jacques. « La vie de Saint Louis et le XIII ${ }^{\mathrm{e}}$ siècle ». Esprit 184 : 38-45.

Le Goff, Jacques. Saint Louis. Paris : Gallimard, 1996.

Le Monde 19 février 1999. « Des Nouvelles de l'Homo biographicus » : XX-XI.

Lepore, Jill. « Historians Who Love Too Much : Reflections on Microhistory and Biography. » JAH 88 (June 2001) : 129-144.

Le Roy Ladurie, Emmanuel. Montaillou, village occitan : de 1294 à 1324. Paris : Gallimard, 1975.

Politix 27 (1994). « La biographie : usages scientifiques et sociaux ». Dir. Luc Berlivet, Annie Collovald et Frédéric Sawicki.

Peneff, Jean. « Les grandes tendances de l'usage des biographies dans la sociologie française ». Politix $27: 25-31$.

Shalhope, Robert E. « Republicanism and Early American Historiography ». WMQ 29 (1952) : 334-356.

Taylor, Allan. William Cooper's Town: Power and Persuasion on the Frontier of the Early American Republic. New York : Vintage, 1995.

Tocqueville, Alexis de. De la démocratie en Amérique. 1835-1840. 2 vols. Paris : Flammarion, 1981.

Valensi, Lucette et Nathan Wachtel. « L'historien errant ». L'Arc 65 (1976) : 3-9

Vesey, Lawrence. «Intellectual History and the New Social History ». Ed. Higham and Conkin. $1-20$.

Viala, Alain. « Racine ou la stratégie du caméléon ». Esprit 184 : 45-53.

Watts, Steven. The Republic Reborn; War and the Making of Liberal America, 1790-1820. Baltimore : The Johns Hopkins UP, 1987.

Wachtel, Nathan. La foi du souvenir : labyrinthes marranes. Paris : Seuil, 2001.

Wiebe, Robert. The Opening of American Society. New York : Knopf, 1984.

Wood, Gordon. «Intellectual History and the Social Sciences ». Ed. Higham and Conkin. 21-38

Wulf, Naomi. « L'idée de démocratie aux États-Unis, de 1828 à 1844, à travers les écrits d'Orestes Brownson ». Thèse nouveau régime, Université Paris 7, décembre 1995.

Young, Alfred H. The Shoemaker and the Tea Party. Boston : Beacon Press, 1999.

Liens 
Johnson, Paul E. « Inheriting the Revolution, by Joyce Appleby ». http://

www.historycooperative.org/journals/wm/58.1/br_12.html

Waldstreicher, David. «Appleby's Liberal America ». www.common-place.org/vol-01/no-01/ reviews/appleby.shtml (vol.1, No.1, September 2000).

\section{NOTES}

1. On retrouve ce même changement d'optique, de la construction théorique vers l'étude d'un destin individuel, quoique toujours assez idéologique, chez l'historien John Patrick Diggins, dans la biographie qu'il a dernièrement consacrée à Lincoln (Diggins). Il nous faut également citer, pour la période révolutionnaire, le tournant opéré dans l'approche historiographique par Alfred Young, l'historien par excellence du radicalisme dans la Révolution américaine, dans sa récente biographie consacrée à un cordonnier révolutionnaire (Young).

\section{RÉSUMÉS}

This paper aims at assessing the relatively recent rediscovery of biography as a historical genre and its place in the historiography of the Early Republic. Beyond biography as a traditional genre, many historians have reinvented a biographical approach to history in which they do not merely tell a life-story, but study individuals as they reveal and reflect the period in which they live. This renewed approach is particularly telling of the early years of the American Republic when a sense of individualism emerged within mass democratic culture.

\section{INDEX}

Mots-clés : biographie, Jeune République, individualisme, historiographie Keywords : Annales, biography, Early Republic, individualism, historiography

\section{AUTEUR}

\section{NAOMI WULF}

Naomi Wulf est maître de conférences à l'Université Paris 12 - Val-de-Marne. 